\title{
Intelligent Diagnosis Method for Centrifugal Pump System Using Vibration Signal and Support Vector Machine
}

\author{
Hongtao Xue, ${ }^{1}$ Zhongxing Li, ${ }^{1}$ Huaqing Wang, ${ }^{2}$ and Peng Chen ${ }^{3}$ \\ ${ }^{1}$ School of Automotive and Traffic Engineering, Jiangsu University, 301 Xuefu Road, Zhenjiang, Jiangsu 212013, China \\ ${ }^{2}$ Beijing University of Chemical Technology, 15 Beisanhuan East Road, Chaoyang, Beijing 100029, China \\ ${ }^{3}$ Graduate School of Bioresources, Mie University, 1577 Kurimamachiya-cho, Tsu, Mie 514-8507, Japan \\ Correspondence should be addressed to Huaqing Wang; wanghq_buct@hotmail.com and Peng Chen; chen@bio.mie-u.ac.jp
}

Received 27 May 2014; Revised 28 August 2014; Accepted 28 August 2014; Published 4 November 2014

Academic Editor: Didier Rémond

Copyright (C) 2014 Hongtao Xue et al. This is an open access article distributed under the Creative Commons Attribution License, which permits unrestricted use, distribution, and reproduction in any medium, provided the original work is properly cited.

\begin{abstract}
This paper proposed an intelligent diagnosis method for a centrifugal pump system using statistic filter, support vector machine (SVM), possibility theory, and Dempster-Shafer theory (DST) on the basis of the vibration signals, to diagnose frequent faults in the centrifugal pump at an early stage, such as cavitation, impeller unbalance, and shaft misalignment. Firstly, statistic filter is used to extract the feature signals of pump faults from the measured vibration signals across an optimum frequency region, and nondimensional symptom parameters (NSPs) are defined to represent the feature signals for distinguishing fault types. Secondly, the optimal classification hyperplane for distinguishing two states is obtained by SVM and NSPs, and its function is defined as synthetic symptom parameter (SSP) in order to increase the diagnosis' sensitivity. Finally, the possibility functions of the SSP are used to construct a sequential fuzzy diagnosis for fault detection and fault-type identification by possibility theory and DST. The proposed method has been applied to detect the faults of the centrifugal pump, and the efficiency of the method has been verified using practical examples.
\end{abstract}

\section{Introduction}

Pumps come in several types such as centrifugal, turbo, propeller, and positive displacement. Irrigation pumping plants are usually of the centrifugal type [1]. A centrifugal pump plays an important role in industries. However, faults of pump can cause a high rate of energy loss associated with performance degradation, even the breakdown of a whole system, and then lead to substantial economic losses. Therefore, condition diagnosis of the pump system at an early stage is very important.

Different approaches have been used for fault detection of centrifugal pumps. Studies in [2-4] adopted indirect parameters or interrelated parameters to identify the faults but did not consider the features of a vibration signature. However, it is gradually being understood that vibration signature is the most revealing information reflecting the condition of rotating machinery [5-7]. Vibration signals were employed for fault detection and condition monitoring in [8-21], so it is important that fault signal should be sensitively extracted from the measured signal when fault occurs. However, it is difficult since fault signal is so weak that it is often buried in strong noise, especially at an early stage. Studies in [814] presented that wavelet analysis could detect effectively condition change. However, different wavelet basis functions were adopted. Moreover, the features of vibration signals were captured in different frequency areas [14]. Therefore, the line between the application of wavelet analysis and the construction of an automatic system is drawn.

For noise cancelling, namely, extracting fault signal, many methods have been proposed. For example, band-pass filter [22], adaptive filter [23], Wiener filter [24] and Kalman filter [25], and so forth. However in the field of machinery diagnosis, these methods cannot always be applied to failure signal extraction, due to the following adverse conditions. Firstly, in the case of the band-pass filter, the wide band noise cannot be cancelled. Secondly, when applying the adaptive filter for noise cancelling, the reference noise must be simultaneously 
measured with the signal. Simultaneous measurement of the reference noise is not easily realized in most cases of fault diagnosis. Finally, the noise cannot be effectively removed by the Wiener filter and Kalman filter if noise and signal do not follow the normal distribution. In order to overcome the problems above, we used the statistic filter to extract the fault signal from the vibration signal measured in the abnormal state of a machine.

Fault diagnosis can hardly do without intelligent systems such as neural networks (NN) and support vector machine (SVM). Studies in [14-16] have been carried out to investigate the use of NN for detecting fault and identifying fault types. However, the conventional NN cannot reflect the possibility of ambiguous diagnosis problems and will never converge when the first layer symptom parameters have the same values in different states [26]. Studies in [17-20] have employed SVMs to perform fault diagnosis, and the capability of SVMs which can efficiently perform a nonlinear classification with kernel function has been confirmed. However, it is difficult to determine the parameters for a given value of the regularization and kernel parameters and to choose an appropriate kernel function.

For the above reasons, in order to process the uncertain relationship between symptom parameters (SPs) and machinery conditions, improve the efficiency and accuracy of fault diagnosis at an early stage, and achieve an automatic system from measured signals, the authors propose an intelligent diagnosis for a pump system using support vector machine (SVM), possibility theory, and Dempster-Shafer theory (DST) on the basis of the vibration signals, to detect faults and identify fault types at an early stage, as is shown in the flowchart in Figure 1. The statistic filter is used to detect and extract the failure signals of pump faults across optimum frequency regions, and the failure signals extracted are reflected by nondimensional symptom parameters (NSPs). In order to increase the diagnosis' sensitivity, a synthetic symptom parameter (SSP) is defined with the function of the optimal classification hyperplane obtained using SVM. Then the possibility distributions of the SSPs are used to detect faults and identify fault types by possibility theory and DST. Finally, the proposed method is evaluated using practical examples of centrifugal pump, which shows a good performance.

\section{Experimental Centrifugal Pump System for Condition Diagnosis}

Figure 2 shows the testing bed consisting of a pump, a motor, and a close-loop water piping system. The SF-JRO series motor is employed to drive the pump through a coupling, and the rotating speed can be controlled via control panel. The experimental pump is a centrifugal pump described as follows: HONDA Pump, Type: HAS (Volute Pump Horizontal Type), Head: 40, Output: $3.7 \mathrm{~kW}$, and Capacity: $7.5 \mathrm{~m}^{3} / \mathrm{h}$. The capacity of the tank is based on the maximum flow rate which can also be controlled by the valve control system. Five accelerometers are adopted to acquire the vibration signals with a sampling frequency of $50 \mathrm{kHz}$. One is mounted on the pump housing, two in the piping direction and vertical

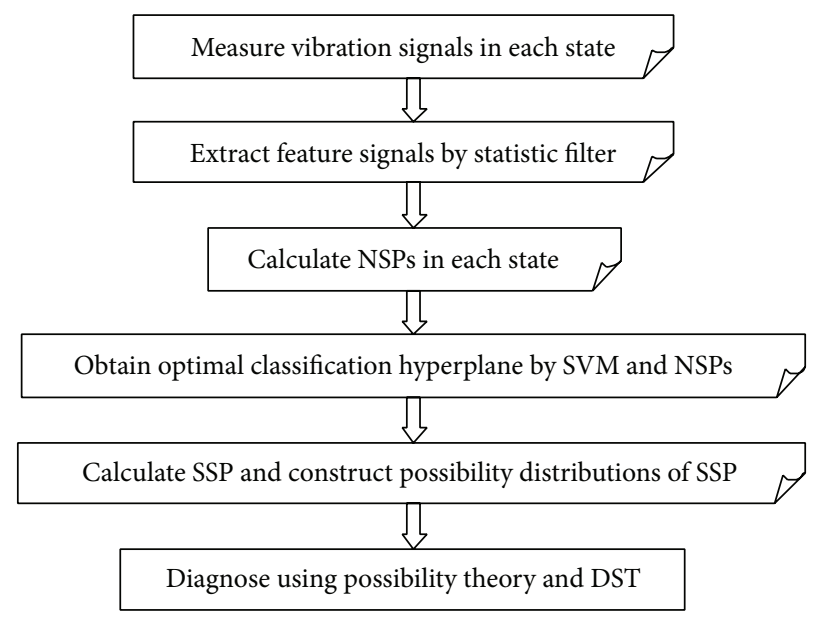

FIgURE 1: Flowchart of intelligent diagnosis.

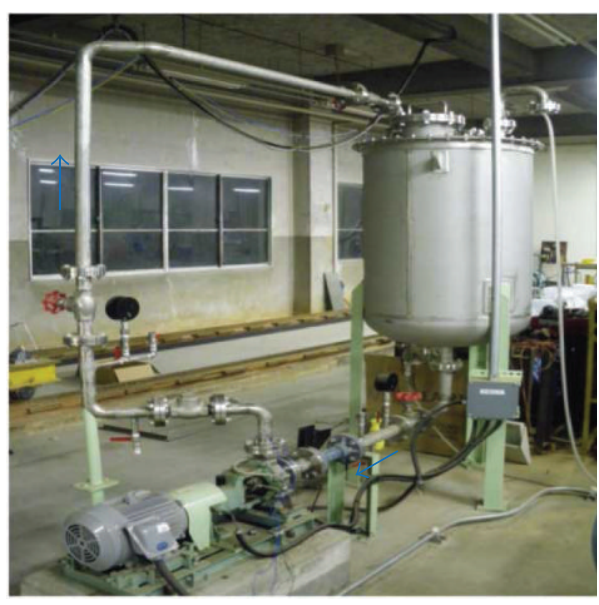

FIgURE 2: Photograph of the experiment centrifugal pump system.

direction of the pump inlet, respectively, the rest in the piping direction and horizontal direction of the pump outlet, as shown in Figure 3.

Cavitation phenomenon is one of the sources of instability in a centrifugal pump. Bubbles collapse abruptly leading to damage of the pump and generation of crackling sound and vibration [27]. Other faults, such as impeller unbalance and shaft misalignment between the motor and the pump, are discriminated easily, but often occur in pump system. These faults can cause serious machine accidents and bring great production losses. Therefore, these states with normal state will be discussed to examine whether incipient faults of a pump were able to be detected using the proposed method in a later section. When the pump speed was at $3500 \mathrm{rpm}$ and the valves in the suction and discharge lines were fully open, the pump was in the best situation of operation. The condition was seen as normal state. Then the conditions of the speed and the valve in the discharge line remained unchanged; the valve in the suction line was slowly turned down until little bubbles appeared. At present the condition was the cavitation 


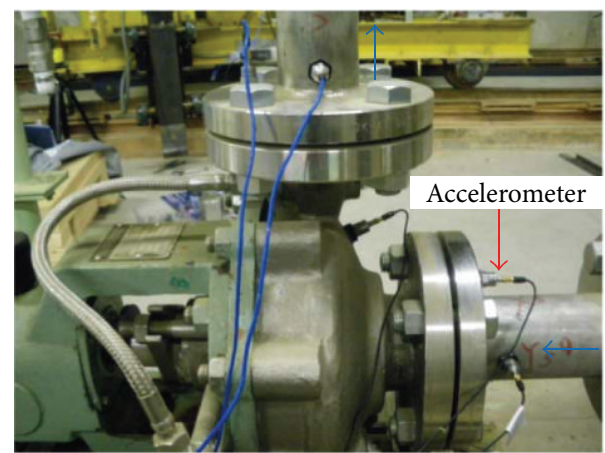

FIgURE 3: The location of sensors.

state with the mild abnormal degree. Sequentially, the valve in suction line was turned down; the amounts of bubbles were used to decide the cavitation state with the medium and severe abnormal degree. In the case of impeller unbalance due to impeller damage, the damage areas from three impellers were $25 \mathrm{~mm}^{2}, 100 \mathrm{~mm}^{2}$, and $225 \mathrm{~mm}^{2}$, respectively. Then the states that used three impellers were defined as impeller unbalance with the mild, medium, and severe abnormal degree. Similarly, the states that the pump side of the shaft was installed to deviate $0.1 \mathrm{~mm}, 0.5 \mathrm{~mm}$, and $1.0 \mathrm{~mm}$ beyond its specified limit were defined as shaft misalignment with different abnormal degrees.

\section{Feature Extraction Using Statistic Filter}

3.1. Statistic Filter. Statistic filter is a filter which takes in a packet and decides whether to accept or reject it on the basis of the statistical approach [28]. In the field of machinery diagnosis, studies in [29] have investigated that the filter based on means of statistical tests of spectrums was convenient and effective for extracting pure failure signals. Here, statistic filter based on distinction index (DI) of spectrums between normal signal and measured signal is proposed, as shown in Figure 4.

Measured normal signal and diagnosis signal can be divided into $N$ parts. Spectrum analysis is performed on each part which generates one spectrum content. Going through all signal parts, the spectrum content $F_{i j}\left(f_{k}\right)$ of the part $j(j=$ $1,2, \ldots, N)$ at the frequency $f_{k}\left(f_{k}=k \cdot f_{\max } / M, k=\right.$ $1,2, \ldots, M)$ can be obtained, where $i$ is the style of signal, such as normal signal $(n)$ and diagnosis signal $(d) ; f_{\max }$ and $M$ are the maximal frequency and the number of spectrum analysis. Thus the spectrum components $F_{n}\left(f_{k}\right)$ and $F_{d}\left(f_{k}\right)$ of $N$ signal parts under normal and diagnosis states at the frequency $f_{k}$ can be denoted in (1), and it is verified that $F_{n}\left(f_{k}\right)$ and $F_{d}\left(f_{k}\right)$ conform to normal distribution, as shown in Figure 5. Consider

$$
\begin{gathered}
F_{n}\left(f_{k}\right)=\left\{F_{n 1}\left(f_{k}\right), F_{n 2}\left(f_{k}\right), \ldots, F_{n N}\left(f_{k}\right)\right\} \\
F_{d}\left(f_{k}\right)=\left\{F_{d 1}\left(f_{k}\right), F_{d 2}\left(f_{k}\right), \ldots, F_{d N}\left(f_{k}\right)\right\} .
\end{gathered}
$$

In order to determine whether there is significant difference between the spectrums $F_{n}\left(f_{k}\right)$ and $F_{d}\left(f_{k}\right)$ of normal and diagnosis states at the frequency $f_{k}$, distinction index (DI) is defined as follows as an index of the quality to evaluate the spectrum difference between normal and diagnosis states. Suppose that $x_{1}$ and $x_{2}$ are the values of $F_{n}\left(f_{k}\right)$ and $F_{d}\left(f_{k}\right)$ and conform to the normal distributions $N\left(\mu_{1}, \sigma_{1}\right)$ and $N\left(\mu_{2}, \sigma_{2}\right)$, respectively. Here, $\mu_{1}, \sigma_{1}$ and $\mu_{2}, \sigma_{2}$ are the mean values and standard deviations of normal and diagnosis states at the frequency $f_{k}$. The larger the value of $\left|x_{1}-x_{2}\right|$ is, the higher the difference of two states will be. Because $z=x_{2}-x_{1}$ is also distributed $N\left(\mu_{2}-\mu_{1}, \sigma_{1}^{2}+\sigma_{1}^{2}\right)$, we can denote the density function of $z$ in (2a). When $\mu_{2} \geq \mu_{1}$, the probability of $z=x_{2}-x_{1}\left(x_{2}<x_{1}\right)$ can be calculated by (2b); of course, we can obtain the same conclusion when $\mu_{2} \leq \mu_{1}$. Standardizing the value of $z$, namely, $\mu=\left(z-\left(\mu_{2}-\mu_{1}\right)\right) / \sqrt{\sigma_{1}^{2}+\sigma_{1}^{2}}$, (2b) can be transformed into (2c). Consider

$$
\begin{gathered}
f(z)=\frac{1}{\sqrt{2 \pi\left(\sigma_{1}^{2}+\sigma_{2}^{2}\right)}} \exp \left(-\frac{\left\{z-\left(\mu_{2}-\mu_{1}\right)\right\}^{2}}{2\left(\sigma_{1}^{2}+\sigma_{2}^{2}\right)}\right), \\
p=\int_{-\infty}^{0} f(z) d z, \\
p=\frac{1}{\sqrt{2 \pi}} \int_{-\infty}^{-\mathrm{DI}} \exp \left(-\frac{\mu^{2}}{2}\right) d \mu .
\end{gathered}
$$

Here, $\mathrm{DI}=\left(\mu_{2}-\mu_{1}\right) / \sqrt{\sigma_{1}^{2}+\sigma_{1}^{2}}$. Considering the condition of $\mu_{2} \leq \mu_{1}$, the DI value is calculated by the following:

$$
\mathrm{DI}=\frac{\left|\mu_{2}-\mu_{1}\right|}{\sqrt{\sigma_{1}^{2}+\sigma_{2}^{2}}} .
$$

According to the nature of the normal distribution, the probability of $z=x_{2}-x_{1}\left(x_{2} \geq x_{1}\right)$ is also $p$; then the probability of $z$ is $2 p$. However, $z$ shows the overlapping part of normal and diagnosis states; the distinction probability of normal state or diagnosis state is $1-2 p$; then the distinction rate $(\mathrm{DR})$ is defined as follows:

$$
\mathrm{DR}=1-2 p .
$$

In [30-32], DI has been used to judge the sensitivity of a symptom parameter (SP), and it also has been proved that the larger the value of the DI, the higher the DR value will be and then the higher the sensitivity of the SP will be. Moreover, when the DI value is more than 1.28 , the DR value is more than 0.9 , but the probability of $z$ is less than 0.1 . In statistics, significance levels, such as $0.1,0.05$, or 0.01 , are used, depending on the field of study. Here, 0.1 level is used to determine whether there is a difference between $F_{n}\left(f_{k}\right)$ and $F_{d}\left(f_{k}\right)$, and then the DI value of 1.28 is watershed in the statistic filter based on distinction index of spectrums. When the DI value of $F_{n}\left(f_{k}\right)$ and $F_{d}\left(f_{k}\right)$ is smaller than 1.28 , there is smaller difference between $F_{n}\left(f_{k}\right)$ and $F_{d}\left(f_{k}\right)$, and then it is assumed no difference. Therefore, the spectrum $F_{d}\left(f_{k}\right)$ of diagnosis state at the frequency $f_{k}$ will be removed as noise by a filter. Otherwise, $F_{d}\left(f_{k}\right)$ are left with no change. For example, the DI value between $F_{n}\left(f_{1}\right)$ and $F_{d}\left(f_{1}\right)$, as shown 


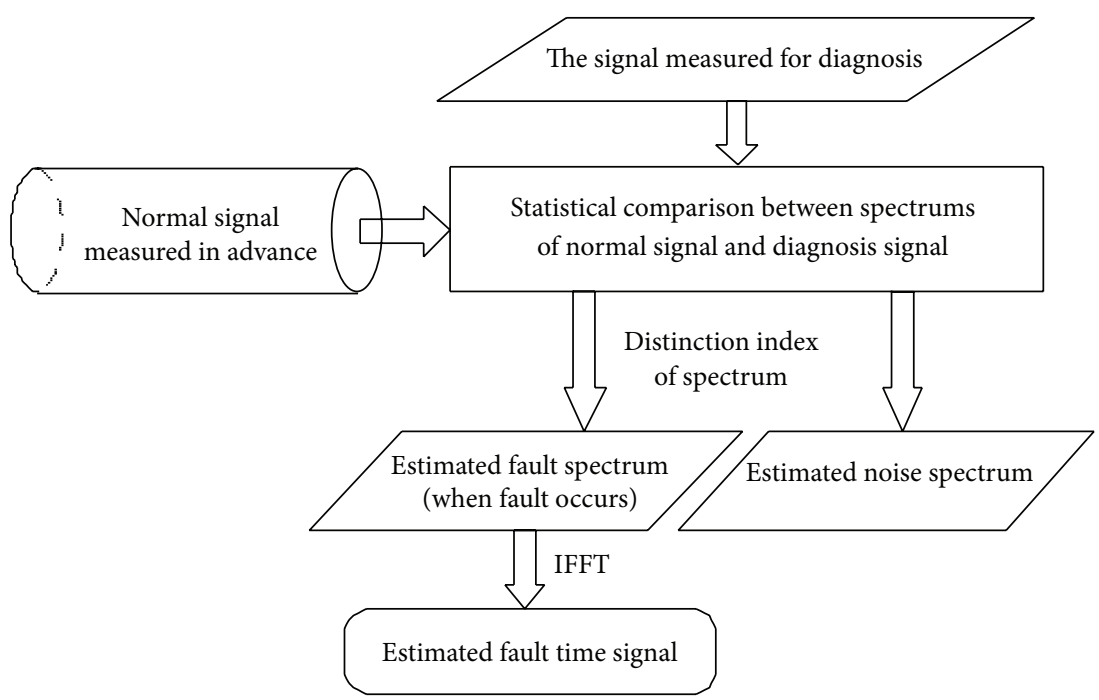

FIGURE 4: The principle of statistic filter based on distinction index (DI) of spectrums.

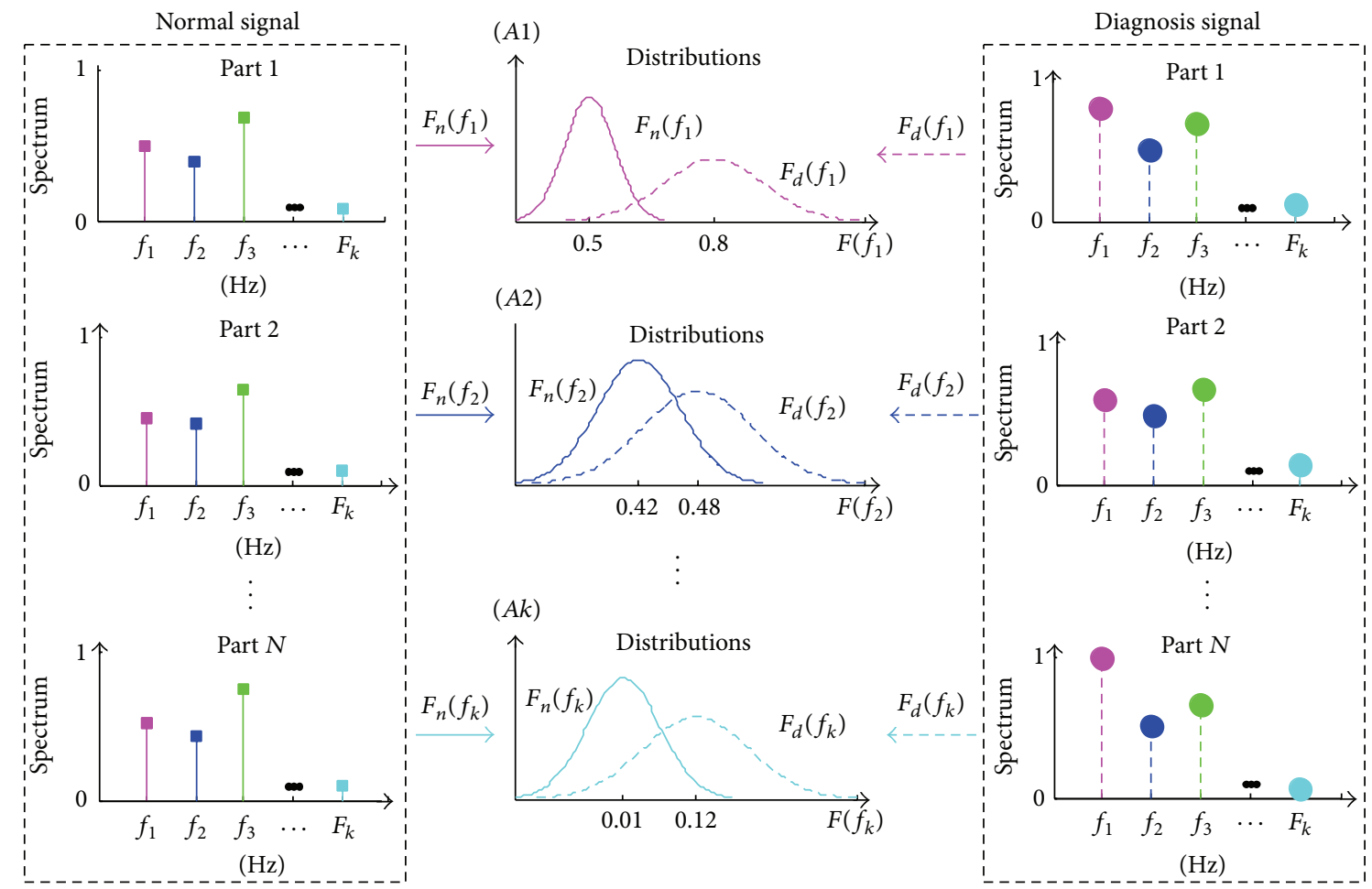

FIGURE 5: Spectrums of each part between normal and diagnosis signals and the normal distributions of $F_{n}\left(f_{k}\right)$ and $F_{d}\left(f_{k}\right)$ at the frequencies $f_{1}$ and $f_{2}$.

in Figure 5(A1), is 1.87; then $F_{d}\left(f_{1}\right)$ is left. However, the DI value between $F_{n}\left(f_{2}\right)$ and $F_{d}\left(f_{2}\right)$, as shown in Figure 5(A2), is 0.79 ; then $F_{d}\left(f_{2}\right)$ is removed from original spectrum. Thus, a filtered $F_{d}\left(f_{k}\right)$ can be obtained, which is controlled by DI. A simple example is introduced to explain a statistic filter based on distinction index (DI) of spectrum, as shown in Figure 6. Here black squares are the spectrum values of normal signal and black dots are the spectrum values of diagnosis signal. In these points, the DI values of spectrums between normal and diagnosis signals at $f_{2}, f_{4}, f_{6}, f_{8}, \ldots, f_{M}$ are smaller than 1.28 ; the DI values at $f_{1}, f_{3}, f_{5}, f_{7}, \ldots$ are more than 1.28 . Thus, the filtered diagnosis spectrums which are indicated by black triangle are estimated as the fault components in frequency domain, and the fault signal in time domain can be obtained by using inverse fast Fourier transform (IFFT).

Moreover, we have tried other values of DI to extract the faults signal by statistic filter for the condition diagnosis of the centrifugal pump system, and the optimum value is still 1.28. 
TABLE 1: DI values and standard deviation of DI values of 3 NSPs between two states from the signals estimated by statistic filter.

\begin{tabular}{lccccccccccccc}
\hline \multirow{2}{*}{ Two states } & \multicolumn{3}{c}{ In mild step } & \multicolumn{4}{c}{ In medium step } & \multicolumn{3}{c}{ In severe step } & \multicolumn{3}{c}{ DI's standard deviation } \\
& $P_{1}$ & $P_{2}$ & $P_{3}$ & $P_{1}$ & $P_{2}$ & $P_{3}$ & $P_{1}$ & $P_{2}$ & $P_{3}$ & $P_{1}$ & $P_{2}$ & $P_{3}$ \\
\hline Normal state and abnormal state & 0.54 & 0.61 & 0.73 & 0.83 & 0.79 & 0.92 & 1.29 & 1.16 & 1.31 & 0.38 & 0.28 & 0.30 \\
Cavitation and impeller unbalance & 0.61 & 0.55 & 0.80 & 0.82 & 0.65 & 0.88 & 1.27 & 1.04 & 0.94 & 0.34 & 0.26 & 0.07 \\
Cavitation and shaft misalignment & 0.58 & 0.64 & 0.72 & 0.76 & 0.90 & 0.87 & 1.03 & 0.98 & 1.26 & 0.23 & 0.18 & 0.28 \\
Impeller unbalance and shaft misalignment & 0.68 & 0.77 & 0.79 & 0.93 & 0.84 & 1.03 & 1.11 & 1.09 & 1.17 & 0.22 & 0.17 & 0.19 \\
\hline
\end{tabular}

TABLE 2: DI values and standard deviation of DI values of 3 NSPs between two states from original signals.

\begin{tabular}{lccccccccccccc}
\hline \multirow{2}{*}{ Two states } & \multicolumn{3}{c}{ In mild step } & \multicolumn{4}{c}{ In medium step } & \multicolumn{3}{c}{ In severe step } & \multicolumn{3}{c}{ DI's standard deviation } \\
& $P_{1}$ & $P_{2}$ & $P_{3}$ & $P_{1}$ & $P_{2}$ & $P_{3}$ & $P_{1}$ & $P_{2}$ & $P_{3}$ & $P_{1}$ & $P_{2}$ & $P_{3}$ \\
\hline Normal state and abnormal state & 0.35 & 0.39 & 0.44 & 0.56 & 0.65 & 0.60 & 0.82 & 0.77 & 0.91 & 0.24 & 0.19 & 0.24 \\
Cavitation and impeller unbalance & 0.33 & 0.20 & 0.53 & 0.45 & 0.41 & 0.59 & 0.88 & 0.75 & 0.72 & 0.29 & 0.28 & 0.10 \\
Cavitation and shaft misalignment & 0.27 & 0.38 & 0.34 & 0.44 & 0.68 & 0.41 & 0.69 & 0.56 & 0.95 & 0.21 & 0.15 & 0.33 \\
Impeller unbalance and shaft misalignment & 0.42 & 0.45 & 0.41 & 0.60 & 0.59 & 0.51 & 0.73 & 0.61 & 0.83 & 0.16 & 0.09 & 0.22 \\
\hline
\end{tabular}

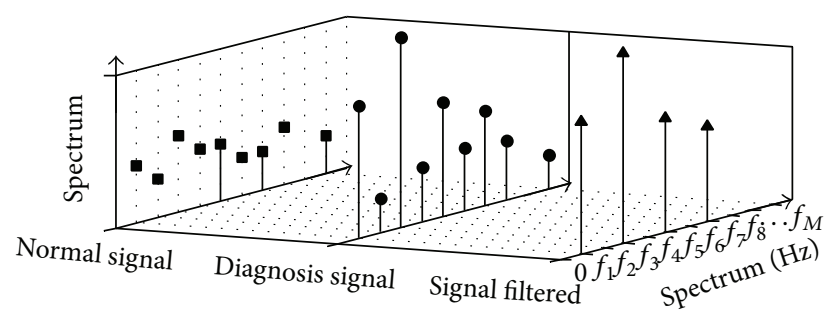

FIGURE 6: Explanation of statistic filter based on distinction index (DI) of spectrums.

3.2. Analysis of Experimental Signals Using Statistic Filter. To make the signals comparable regardless of differences in magnitude, the signals of each state are normalized by the following formula:

$$
x_{i}=\frac{x_{i}^{\prime}-\mu^{\prime}}{\sigma^{\prime}},
$$

where $x_{i}^{\prime}(i=1,2, \ldots, N)$ are the original signal series, $\mu^{\prime}$ and $\sigma^{\prime}$ are the mean and standard deviation of $x_{i}^{\prime}$, and $x_{i}(i=$ $1,2, \ldots, N)$ are the normalization signal series.

In present work, the normalized signal of each state has been divided into 128 parts, respectively. Then statistic filter based on distinction index of spectrum is performed to obtain the estimated signals under different states. Figure 7 shows the estimated signal in time domain of shaft misalignment when the failure is sever. It is obvious that information representing shaft misalignment has been extracted.

\section{Nondimensional Symptom Parameters for Intelligent Diagnosis}

For intelligent diagnosis, symptom parameters (SPs) are required and can sensitively detect the occurrence of fault and distinguish the fault types. A large set of symptom parameters has been defined in the pattern recognition field [31]. Here, the nondimensional symptom parameters (NSPs) in time domain, commonly used for the fault diagnosis of plant machinery, are considered.

When given the digital data $x_{i}(i=1,2, \ldots, N)$ of the vibration signal, where $N$ is the number of the signal, the 3 NSPs in the time domain are defined as follows:

$$
\begin{gathered}
P_{1}=\frac{\sigma}{\overline{|x|}} \\
P_{2}=\frac{\sum_{i=1}^{N}\left(x_{i}-\bar{x}\right)^{3}}{N \cdot \sigma^{3}} \\
P_{3}=\frac{\sum_{i=1}^{N}\left(x_{i}-\bar{x}\right)^{4}}{N \cdot \sigma^{4}},
\end{gathered}
$$

where $\bar{x}$ and $\sigma$ are the mean value and standard deviation of the vibration signal $x_{i}$. $\left|x_{i}\right|$ is the absolute value of the vibration signal $x_{i}$ and $\overline{|x|}$ is the mean value of $\left|x_{i}\right|$.

Then the estimated signals obtained in Section 3 using statistic filter are performed to calculate 3 NSPs. In this paper, 3 typical faults have been investigated with different abnormal degrees in experiment, and then these fault states with the same abnormal degree are called abnormal state with the same abnormal degree for purposes of discussion. Thus, 3 NSPs of these fault states with the same abnormal degree are united to 3 NSPs of abnormal state with the same abnormal degree. Therefore, the DI values of 3 NSPs between any two states in the same abnormal degree are calculated, as shown in Table 1. Moreover, original signals are also done with the same work and DI values of which are shown in Table 2.

It is obvious that the DI values of 3 NSPs from the signals estimated by statistic filter are larger than from original signals. However, the DI values of these SPs are yet low, especially, at an incipient stage of a fault. Thus the choice of an effective SP will expend time and effort, and the differentiation degree of the SP may or may not be high. In this study, a new method in which some SPs integrate an effective SP for diagnosis by introducing weight coefficient is proposed. Here, 


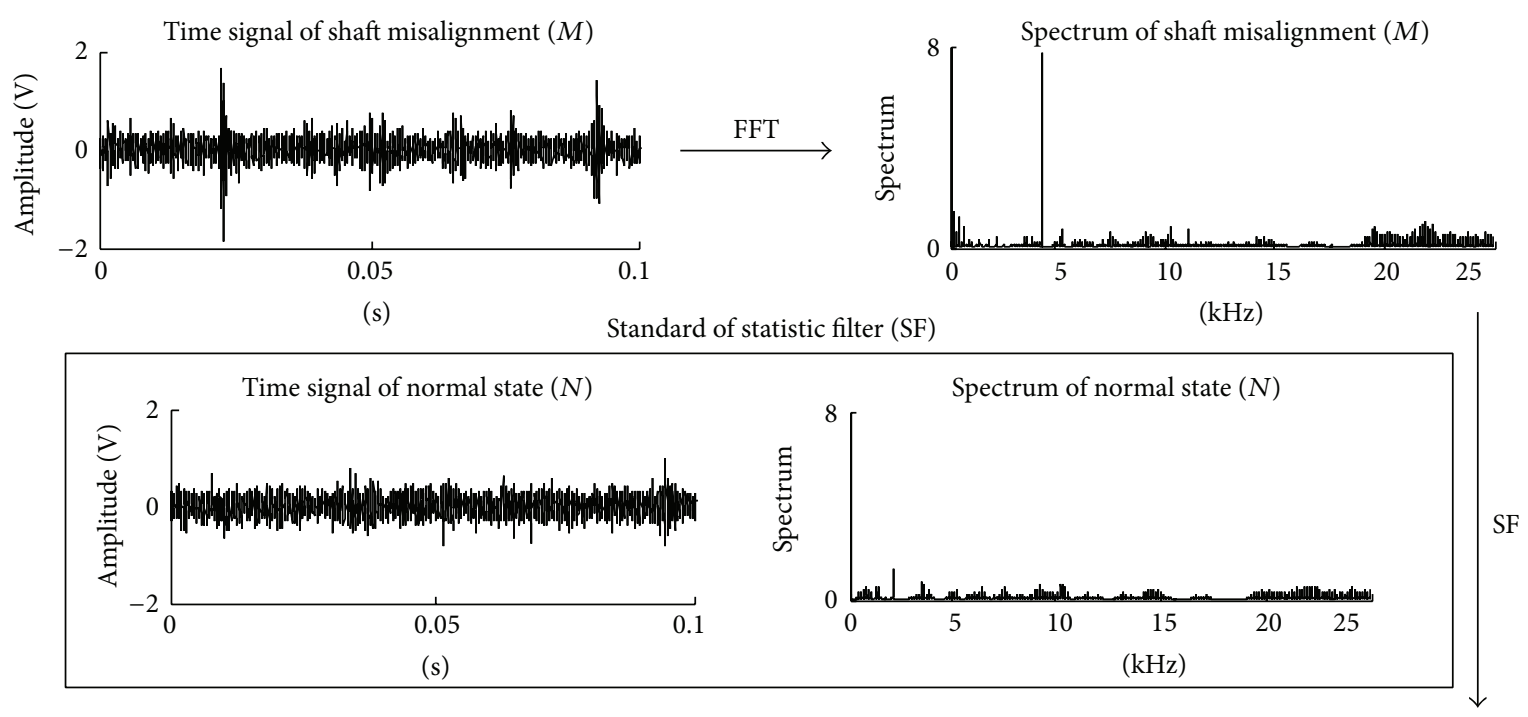

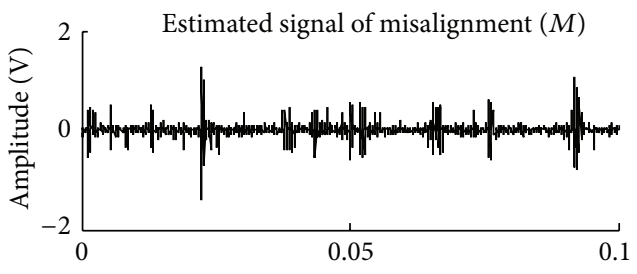

(s)

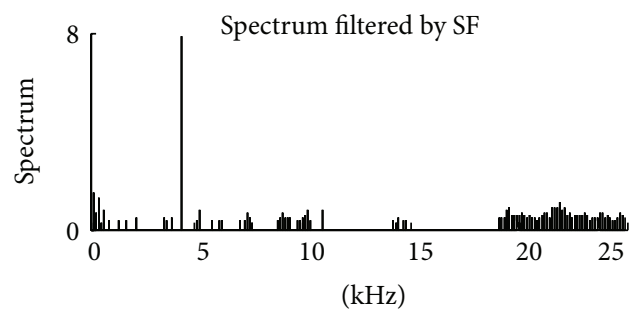

FIGURE 7: Estimated signal of shaft misalignment in sever step using statistic filter based on distinction index of spectrum.

3 NSPs are used to integrate an effective SP, and this integrated SP is called "synthetic symptom parameter (SSP)."

\section{Application of SVM for Synthetic Symptom Parameter}

5.1. Support Vector Machine (SVM). SVM is a relatively new computational learning method based on the statistical learning theory, and the basic idea is to create an optimal classification hyperplane between the two classes and ensure that the distance between the boundary and the nearest data point in each class is maximized [33]. SVM can efficiently perform not only a linear classification but also a nonlinear classification using kernel function. In general, a classification hyperplane can be expressed as follows:

$$
\omega \cdot x+b=0
$$

where $x$ is the sample vector, $\omega$ is a vector that represented the weight coefficients of $x$, and $b$ is a classification threshold.

Recently, almost all of practical applications of SVMs have employed different kernel functions, such as polynomial kernel and radial basis function (RBF) kernel, to achieve linear classifications in high-dimensional feature spaces. However, as said in introduction section, it is difficult to determine the parameters for a given value of the regularization and kernel parameters and to choose an appropriate kernel function. But soft margin SVM was proposed to increase the enchantment of SVM [34]. Soft margin SVM is a modified maximum

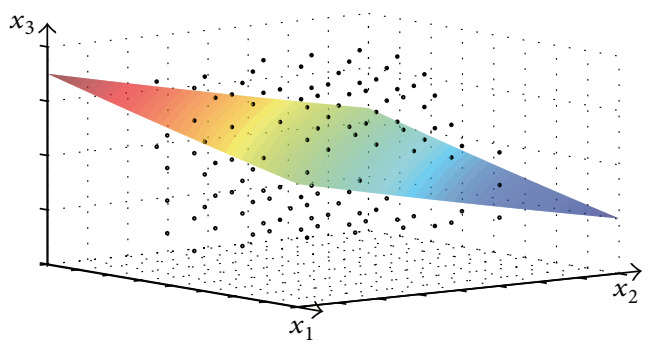

Figure 8: The optimal classification hyperplane for identifying two states using soft margin SVM.

margin idea that permits minimum error and relaxes the condition for the optimal classification hyperplane. In order to explain the soft margin SVM, an example is shown in Figure 8 with 3-dimensional situation. The white circles represent state 1 and the black points represent state 2. $x_{1}, x_{2}$, and $x_{3}$ represent 3 -dimensional sample vector. The colorized plane is the optimal classification hyperplane.

Soft margin SVM not only constructs the optimal classification hyperplane when nonlinear data is separated, but also has a strong generalization capacity when only a small amount of training samples are available.

In order to distinguish states of machinery as precisely as possible, the excellent symptom parameters must be defined for the automatic diagnosis. The symptom parameters must be able to sensitively reflect the characteristics of states. 
However, there is not an acceptable method for extracting symptom parameters from signals measured in each state $[35,36]$. The larger the number of states there is, the more difficult the extraction of symptom parameters is. In many cases, this has been done merely by trial and error. The above process not only is time-consuming and labor intensive, but also cannot always ensure that excellent symptom parameters will be found.

In order to resolve this problem, we propose the new method be called "sequential diagnosis using synthetic symptom parameters generated by soft margin SVM." By this method, the optimum synthetic symptom parameters for distinguishing all states can be sequentially and quickly searched out. Because the synthetic symptom parameters generated by the soft margin SVM can only be used to distinguish tow states, we will sequentially and precisely diagnose states as shown Figure 11.

5.2. Synthetic Symptom Parameter. The paper proposes soft margin SVM to achieve the optimization of integrating primitive SPs in the field of condition diagnosis. The optimal classification hyperplane is shown in (5), where $\omega$ is the optimal vector of the weight coefficients and determines the diagnostic sensitivity of the SSP. Then SSP is defined as follows:

$$
\mathrm{SSP}=\omega \cdot\left[\begin{array}{llll}
P_{1} & P_{2} & \cdots & P_{n}
\end{array}\right]^{\prime}+b .
$$

In order to enhance the sensitivity of extracted features to failures, especially at the incipient stage of a fault, 3 NSPs of each state are input SVMs to train the classifiers. For example, in the first step, the diagnosis goal is to detect whether there is fault, so these two classes are normal and abnormal states. In a normal state, 3 NSPs $x_{i}=\left\{P_{1 i}, P_{2 i}, P_{3 i}\right\}(i=1,2, \ldots, I)$ are extracted to associate with labels $y_{i}=1,(i=1,2, \ldots, I)$ and to recombine the training data $\left(x_{i}, y_{i}\right)(i=1,2, \ldots, I)$ for a normal state. Similarly, the training data of abnormal state $\left(x_{j}, y_{j}\right)(j=1,2, \ldots, J)$ are obtained to associate with labels $y_{j}=-1,(j=1,2, \ldots, J)$. Here, $I$ and $J$ are the class number of 3 NSPs from normal and abnormal states. When these data are input into SVMs to train for the optimal classification hyperplane, shown as Figure 8, weight coefficient $\omega=\left\{\omega_{1}, \omega_{2}\right.$, $\left.\omega_{3}\right\}$ and classification threshold $b$ are obtained, and then the SSPs are obtained, shown in Table 3. Then new SSPs between two faults are calculated based on the estimated signals, and the DI values of new SSPs are also calculated as shown in Table 4 . The DI values of SSPs are larger than the DI values of 3 NSPs. In addition, the viewpoints about training performance are as follows.

(1) Soft margin SVM is a modified maximum margin idea that permits minimum error and relaxes the condition for the optimal classification hyperplane. Therefore, it is not important to give the training performance.

(2) Training data are input into soft margin SVM only to build the optimal classification hyperplane. In this paper, the goal is only to define synthetic symptom parameter (SSP).
TABLE 3: Synthetic symptom parameters (SSPs) between two states.

\begin{tabular}{lc}
\hline Two states & \multicolumn{1}{c}{ Synthetic symptom parameter (SSP) } \\
$\begin{array}{l}\text { Normal state and } \\
\text { abnormal state }\end{array}$ & $\mathrm{SSP}_{1}=-3.587 P_{1}+6.109 P_{2}-2.234 P_{3}+10.006$ \\
\hline $\begin{array}{l}\text { Cavitation and } \\
\text { impeller unbalance }\end{array}$ & $\mathrm{SSP}_{2}=0.164 P_{1}+0.897 P_{2}-3.220 P_{3}-0.107$ \\
\hline $\begin{array}{l}\text { Cavitation and } \\
\text { shaft misalignment }\end{array}$ & $\mathrm{SSP}_{3}=-0.593 P_{1}+0.031 P_{2}+1.071 P_{3}+1.934$ \\
$\begin{array}{l}\text { Impeller unbalance } \\
\text { and shaft } \\
\text { misalignment }\end{array}$ & $\mathrm{SSP}_{4}=1.462 P_{1}-2.843 P_{2}-4.566 P_{3}+2.006$ \\
\hline
\end{tabular}

TABLE 4: DI values of new SSPs in the same abnormal degree from the estimated signals.

\begin{tabular}{lccc}
\hline Two states & $\begin{array}{c}\text { In mild } \\
\text { step }\end{array}$ & $\begin{array}{c}\text { In medium } \\
\text { step }\end{array}$ & $\begin{array}{c}\text { In severe } \\
\text { step }\end{array}$ \\
\hline $\begin{array}{l}\text { Normal state and abnormal } \\
\text { state }\end{array}$ & 1.28 & 1.57 & 1.72 \\
$\begin{array}{l}\text { Cavitation and impeller } \\
\text { unbalance }\end{array}$ & 1.09 & 1.26 & 1.54 \\
$\begin{array}{l}\text { Cavitation and shaft } \\
\text { misalignment }\end{array}$ & 0.98 & 1.29 & 1.47 \\
$\begin{array}{l}\text { Impeller unbalance and shaft } \\
\text { misalignment }\end{array}$ & 1.17 & 1.38 & 1.56 \\
\hline
\end{tabular}

\section{Sequential Fuzzy Diagnosis Using Possibility Theory and Dempster-Shafer Theory}

6.1. Possibility Theory. Possibility theory is a mathematical theory for dealing with certain types of uncertainty and an alternative to probability theory. Professor Zadeh first introduced possibility theory in 1978 as an extension of his theory of fuzzy sets and fuzzy logic [37]. Dubois and Prade further contributed to its development [38]. Recently, possibility theory has been used for fault diagnosis $[14,39,40]$. In [14, $39,40]$, possibility theory was applied to condition diagnosis in rotating machinery under varying rotating speeds to process the uncertain relationship between the symptoms and fault types. In the paper, for increasing the diagnosis' sensitivity and improving the identification of ambiguous state, possibility theory is used to construct the system of sequential fuzzy diagnosis.

For fuzzy inference, the membership function of a SP is necessary $[14,39,40]$, which can be obtained from probability density functions of the SP using possibility theory. In this paper, synthetic symptom parameter (SSP) is selected as the features for fault identification, and it is verified that a SSP follows the Weibull distribution. Then probability density functions of a SSP $(f(x))$ can be changed to possibility function $(p(x))$ by the following formulae:

$$
p\left(x_{i}\right)=\sum_{k=1}^{N} \min \left\{\lambda_{i}, \lambda_{k}\right\}, \quad(i, k=1,2, \ldots, N) .
$$


$\lambda_{i}$ and $\lambda_{k}$ can be calculated as follows:

$$
\begin{gathered}
\lambda_{i}=\int_{x_{i-1}}^{x_{i}} \frac{m}{\eta} \cdot\left(\frac{x-x_{0}}{\eta}\right)^{m-1} \exp \left\{-\frac{\left(x-x_{0}\right)^{m}}{\eta}\right\} d x, \\
x_{i}=\bar{x}+\frac{6 i-3 N}{N} \cdot \sigma \\
\lambda_{k}=\int_{x_{k-1}}^{x_{k}} \frac{m}{\eta} \cdot\left(\frac{x-x_{0}}{\eta}\right)^{m-1} \exp \left\{-\frac{\left(x-x_{0}\right)^{m}}{\eta}\right\} d x, \\
x_{k}=\bar{x}+\frac{6 k-3 N}{N} \cdot \sigma,
\end{gathered}
$$

where $N$ is the division number of the domain of the SSP, namely, $[\bar{x}-3 \sigma, \bar{x}+3 \sigma], \bar{x}$ and $\sigma$ are the mean and the standard deviation of the SSP, respectively. $m, \eta$, and $x_{0}$ are the parameters of the shape, scale, and location. Figure 9 shows an illustration of the possibility function and the probability density function.

To identify machinery condition, the membership function is constructed based on the possibility function of the SSP for diagnosis using possibility theory. Figure 10 shows the matching examples of possibility function. The common area, between the possibility functions $\left(p_{i}(x), p_{j}(x)\right)$ of model states $i, j$ and the possibility function $\left(p_{t}(x)\right)$ of diagnostic state $t$, is calculated by the following formula:

$$
\begin{array}{r}
S_{k}=\int_{\overline{x_{t}}-3 \sigma_{t}}^{\overline{x_{t}}+3 \sigma_{t}} \psi_{k}(x) d x, \\
\psi_{k}(x)=\min \left\{p_{k}(x), p_{t}(x)\right\}, \\
(k=i, j),
\end{array}
$$

where $\overline{x_{t}}$ and $\sigma_{t}$ are the mean and the standard deviation of the SSP in diagnostic state. Moreover, the unknown state except state $i$ and state $j$ is also model state UN, its possibility function $\left(p_{\mathrm{UN}}(x)\right)$ is calculated by the following formula (10). And the common area between $p_{\mathrm{UN}}(x)$ and $p_{t}(x)$ is calculated by the following formula (11). Consider

$$
\begin{gathered}
p_{\mathrm{UN}}(x)=\max \left\{1-\left[p_{i}(x)+p_{j}(x)\right], 0\right\}, \\
S_{\mathrm{UN}}=\int_{\overline{x_{t}}-3 \sigma_{t}}^{\overline{x_{t}}+3 \sigma_{t}} \psi_{\mathrm{UN}}(x) d x \\
\psi_{\mathrm{UN}}(x)=\min \left\{p_{\mathrm{UN}}(x), p_{t}(x)\right\}
\end{gathered}
$$

Therefore, the probability that should be diagnosed with model states $i, j$, and $\mathrm{UN}$, respectively, can be calculated by the following formulas. Then it is judged that the diagnostic state is the model state with the largest possibility. Consider

$$
\begin{gathered}
\omega_{k}=\frac{1}{\varepsilon} \cdot \frac{S_{k}}{\int p_{k}(x) d x}, \quad(k=i, j, \mathrm{UN}) \\
\varepsilon=\frac{S_{i}}{\int p_{i}(x) d x}+\frac{S_{j}}{\int p_{j}(x) d x}+\frac{S_{\mathrm{UN}}}{\int p_{\mathrm{UN}}(x) d x} .
\end{gathered}
$$

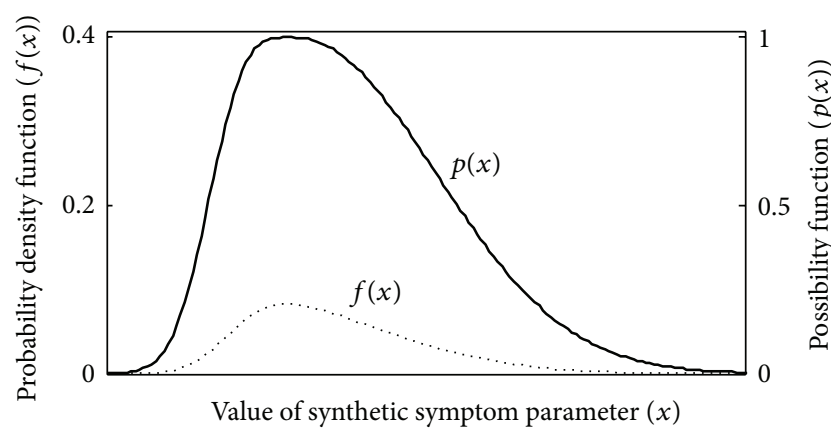

FIGURE 9: Probability density function $(f(x))$ and possibility function $(p(x))$.

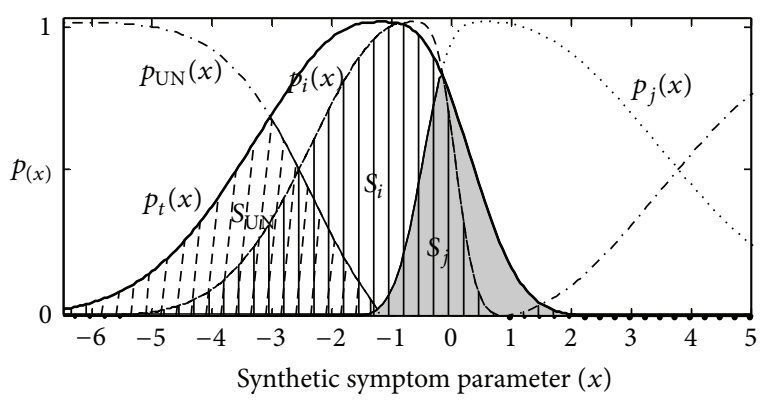

FIgURE 10: Matching example of possibility functions between model states and diagnostic state.

6.2. Sequential Condition Diagnosis Approach. Symptom parameters (SPS) can represent the features of vibration signals. However they are always sensitive to one or several failure types, so it is very difficult to find one or more SPs that simultaneously identify all states, but in contrast it is easy that one or some SPs are found to identify two states. Then a sequential diagnosis method, addressing the multiple failure type identification, as shown in Figure 11, is proposed for condition diagnosis of a centrifugal pump.

The first step of the diagnostic system is simple diagnosis. Its performance is to detect whether there is fault, and then normal state $(N)$ and abnormal state $(A)$ are model states. In order to generalize the capability of condition diagnosis, all $\mathrm{SSP}_{1} \mathrm{~s}$ of each abnormal state with three abnormal degrees (mild, medium, and severe) are combined to establish the model possibility function. Since there are only three typical faults of the pump in this paper, the unknown state except normal state and abnormal state is also a model state. If the possibility grades of normal state $(N)$, abnormal state $(A)$, and the unknown state (UN) are expressed as $\omega(N), \omega(A)$, and $\omega(\mathrm{UN})$, respectively, and $\omega(N)$ is the largest; then the state is diagnosed as "normal state $(N)$," ending with the stop of the diagnosis system. Otherwise, there is fault; the diagnostic system will come into the next step of precise diagnosis.

Precise diagnosis is performed to identify the fault types. Since there are three types of faults in the experiment, three patterns are piled up in the precise diagnosis phase. In each pattern, two fault types are selected as diagnosis target, 


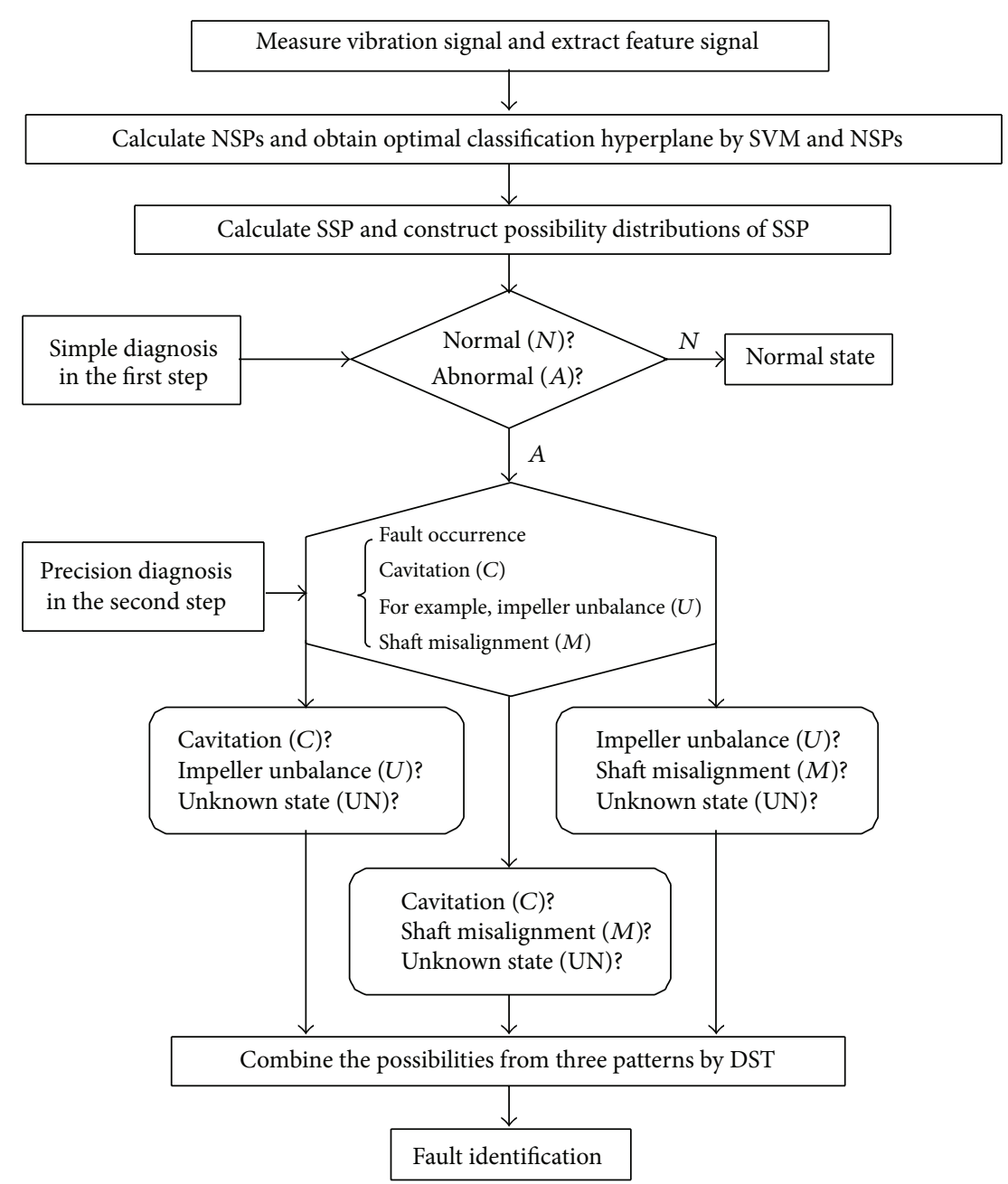

FIGURE 11: Flowchart of sequential diagnosis for a pump system.

and then the unknown state except the two selected states is also regarded as diagnosis target. Thus, there are three states in each pattern. For example, in the first pattern, three model states are cavitation $(C)$, impeller unbalance $(U)$, and unknown state (UN), and then these possibility grades of all $\mathrm{SSP}_{2} \mathrm{~S}$ are expressed as $\omega_{1}(C), \omega_{1}(U)$, and $\omega_{1}(\mathrm{UN})$, respectively. In the second pattern, cavitation $(C)$, shaft misalignment $(M)$, and unknown state (UN) are three model states, and these possibility grades of all $\mathrm{SSP}_{3} \mathrm{~s}$ are expressed as $\omega_{2}(C), \omega_{2}(M)$, and $\omega_{2}(\mathrm{UN})$, respectively. In the third pattern, impeller unbalance $(U)$, shaft misalignment $(M)$, and unknown state (UN) are three model states, and these possibility grades of all $\mathrm{SSP}_{4} \mathrm{~s}$ are expressed as $\omega_{3}(U), \omega_{3}(M)$, and $\omega_{3}(\mathrm{UN})$, respectively.

When the training data, synthetic symptom parameters (SSPs) between two states calculated using the proposed method, are trained to determine the membership function of fuzzy inference, the system of sequential fuzzy diagnosis as shown in Figure 11 has been built.

6.3. Fuzzy Inference Using Dempster-Shafer Theory. Dempster-Shafer theory (DST) is an effective method that combines accumulative evidences [41, 42]. If $N$ evidences are used to identify $M$ classes $\left(A_{1}, A_{2}, \ldots, A_{M}\right)$, and $\omega_{i}$ is the possibility assignment function from the $i$ th $(i=1,2, \ldots, N)$ evidence, the combining possibility function $\omega(A)$ can be obtained by DST, given as follows:

$$
\begin{gathered}
\omega(A)=\frac{1}{k} \sum_{\cap_{i=1}^{N} A_{i}=A} \prod_{i=1}^{N} \omega_{i}\left(A_{i}\right) \\
k=\sum_{\cap_{i=1}^{N} A_{i} \neq \emptyset} \prod_{i=1}^{N} \omega_{i}\left(A_{i}\right) .
\end{gathered}
$$

DST is widely used in the classification to address the uncertainty problem [43-45]. In the Section 6.2, three patterns are simultaneously performed in the second step. Obviously, the inferences are independent in three patterns, and each pattern offers one evidence for obtaining the final identification result. Then Dempster-Shafer theory (DST) is employed to combine the possibility of each fault state. If the possibility grades of cavitation $(C)$, impeller unbalance $(U)$, shaft misalignment $(M)$, and unknown state (UN) in the second step are $\omega(C), \omega(U), \omega(M)$, and $\omega(\mathrm{UN})$, respectively, 
then the possibility grade of each fault state is combined by DST as follows:

$$
\begin{gathered}
\omega(C)=\frac{1}{k} \cdot \omega_{1}(C) \omega_{2}(C) \omega_{3}(\mathrm{UN}) \\
\omega(U)=\frac{1}{k} \cdot \omega_{1}(U) \omega_{2}(\mathrm{UN}) \omega_{3}(U) \\
\omega(M)=\frac{1}{k} \cdot \omega_{1}(\mathrm{UN}) \omega_{2}(M) \omega_{3}(M) \\
\omega(\mathrm{UN})=\frac{1}{k} \cdot \omega_{1}(\mathrm{UN}) \omega_{2}(\mathrm{UN}) \omega_{3}(\mathrm{UN})
\end{gathered}
$$

where $k$ is a normalizing constant, $k=\omega_{1}(C) \omega_{2}(C) \omega_{3}(\mathrm{UN})+$ $\omega_{1}(U) \omega_{2}(\mathrm{UN}) \omega_{3}(U)+\omega_{1}(\mathrm{UN}) \omega_{2}(M) \omega_{3}(M)+\omega_{1}(\mathrm{UN}) \omega_{2}$ $(\mathrm{UN}) \omega_{3}(\mathrm{UN})$. If $\max \{\omega(C), \omega(U), \omega(M), \omega(\mathrm{UN})\}=\omega(C)$, then the diagnosed state is judged as "cavitation $(C)$." In other words, the state that the possibility grade is the biggest is the diagnosed state.

\section{Diagnosis and Verification}

7.1. Diagnosis by the Proposed Method. To verify the proposed diagnostic method, the test data sets are again acquired in accordance with three typical faults of a centrifugal pump and the defect degrees of mild, medium, and severe. Moreover, each data set is processed using the proposed methods as input of the diagnosis system. Here, two practical diagnostic examples are shown in Figures 12 and 13.

In the first example, possibility functions between test state and model states in the first step are shown in Figure 12. $P_{N}(x)$ and $P_{A}(x)$ express the possibility functions of normal state and abnormal state (it includes only cavitation, impeller unbalance, and shaft misalignment in this paper). $P_{\mathrm{UN}}(x)$ expresses the possibility function of the unknown state except normal state and abnormal state. $P_{t}(x)$ expresses the possibility function of the test state. $S_{N}, S_{A}$, and $S_{\mathrm{UN}}$ express the common area between $P_{t}(x)$ and $P_{N}(x), P_{A}(x)$, and $P_{\mathrm{UN}}(x)$, respectively. $\omega(N), \omega(A)$, and $\omega(\mathrm{UN})$, the possibility of normal state, abnormal state, and the unknown state, respectively, are calculated as $0.8558,0.0724$, and 0.0718 . Thus the diagnosis process stops in the first step, and the outcome of diagnosis system is normal state. The diagnosis state is entirely consistent with the original state.

For the second example, the outcome of the first step as shown in Figure 13(a) is abnormal state, and then the diagnostic system comes into the second step of precision diagnosis. In the second step, three patterns are simultaneously performed as shown in Figures 13(b), 13(c), and 13(d). The diagnosis possibilities are shown in Table 5. Moreover, the diagnosis system intelligently employs DST to combine the possibility grade of each fault state. Then $\omega(C), \omega(U), \omega(M)$, and $\omega(\mathrm{UN})$, the possibility of the cavitation $(C)$, impeller unbalance $(U)$, shaft misalignment $(M)$, and unknown state (UN), respectively, are calculated as 0.9649, 0.0001, 0.0098, and 0.0252 . So the final outcome is cavitation state. By comparing the original state, the diagnosis result is correct.

Similarly, 10 test data sets have been performed in the same procedure, and the diagnosis results are shown in

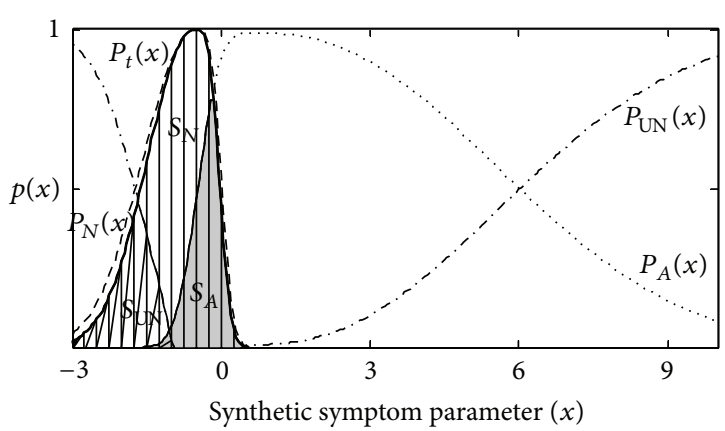

FIGURE 12: The first diagnostic example using the sequential diagnosis proposed.

Table 6. The experimental result has shown that all test states have been diagnosed correctly, but even the faults with mild defect at the early stage have been detected sensitively and identified rightly.

7.2. Diagnosis by Fuzzy Neural Network. Fuzzy neural network is often employed to detect fault and identify fault types [14-16]. This section shows the performance of the diagnosis system based on fuzzy neural network. In general, there are two methods of direct diagnosis and sequential diagnosis, as shown in Figures 14 and 15. Certainly, considering the experiment in the paper, state $i$ means normal state, cavitation, impeller unbalance, or shaft misalignment, sequentially.

To compare the proposed diagnosis with the diagnosis methods based on fuzzy neural network, the experiment data described in Section 2 are used to train the diagnosis systems based on fuzzy neural network as shown in Figures 14 and 15; the measured data described in Section 7 are used to test. The diagnosis results are shown in Table 7. The direct diagnosis based on fuzzy neural network could hardly detect the faults with mild degree, and 2 test data sets with medium degree have been also identified mistakenly. For sequential diagnosis, 6 test data sets with medium and severe degrees have been diagnosed correctly, but the performance that the faults with mild degree will be detected and identified is weak.

\section{Conclusions}

To effectively detect faults and correctly identify fault types for plant machinery at an early stage, an intelligent diagnosis method using statistic filter, SVM, possibility theory, and DST on the basis of the vibration signals was proposed for a centrifugal pump system, and the effectiveness was demonstrated experimentally. The superiority of the method proposed in this paper can be explained in the following points.

(1) Statistic filter is a method of signal processing that failure signal is extracted by statistical tests of spectrums between normal signal and fault signal. The application of statistic filter is very effective, even if at an early stage, statistic filter can extract effectively pure feature signal. 


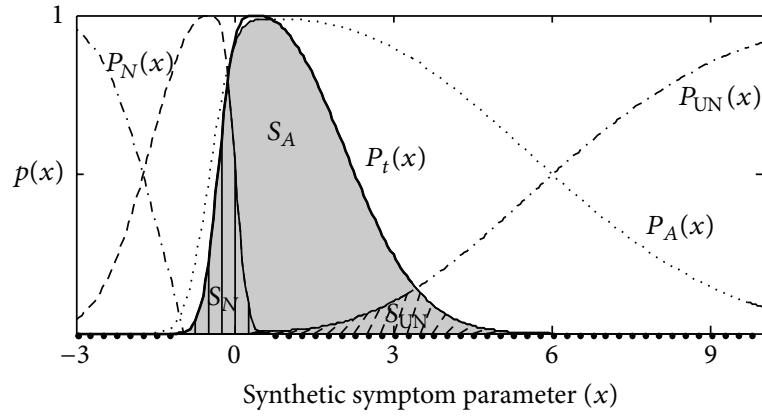

(a)

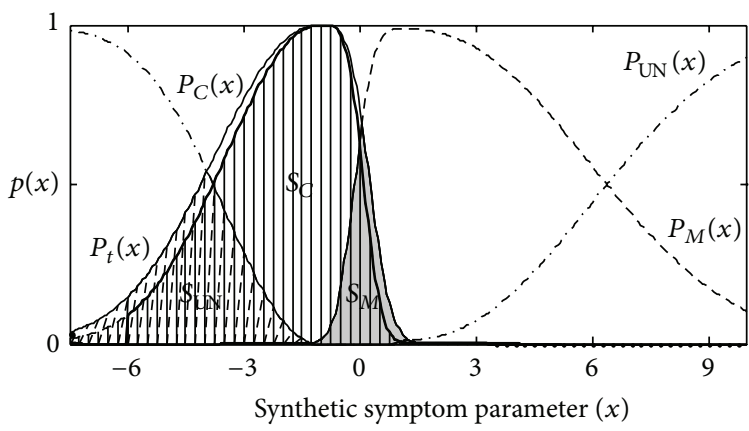

(c)

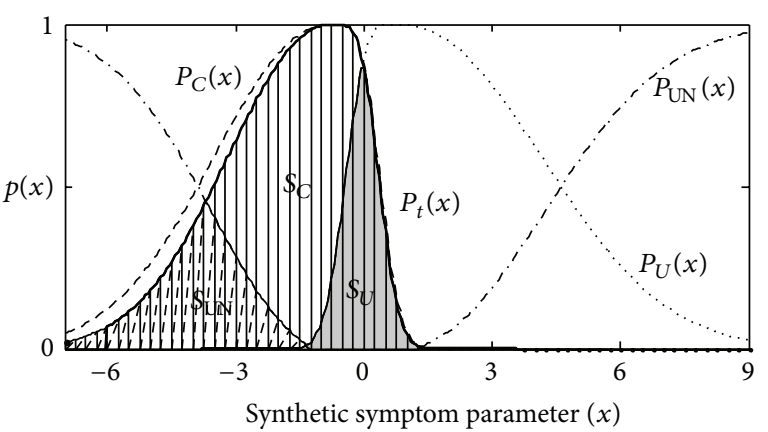

(b)

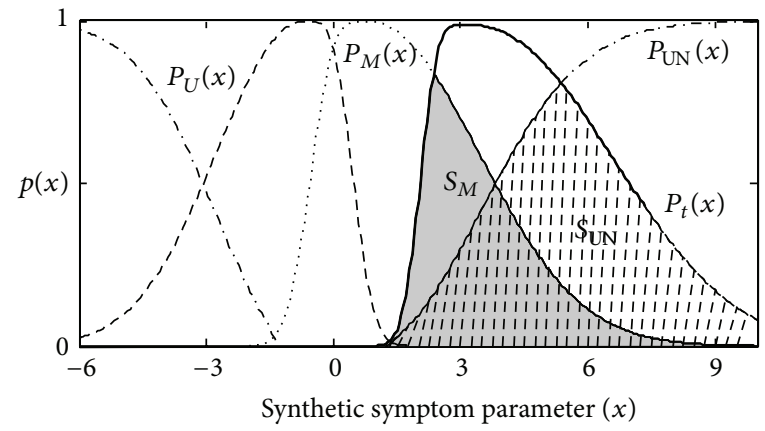

(d)

FIGURE 13: The second diagnostic example using the sequential diagnosis proposed.

TABle 5: The second diagnostic possibilities using the sequential diagnosis proposed.

\begin{tabular}{lccr}
\hline Each step or pattern & Model state 1 & $\begin{array}{c}\text { Possibility of model state } \\
\text { Model state 2 }\end{array}$ & Model state 3 \\
\hline $\begin{array}{l}\text { In the first step } \\
\text { In the second step }\end{array}$ & $\omega(N)=0.3200$ & $\omega(A)=0.6134$ & $\omega(\mathrm{UN})=0.0666$ \\
$\quad$ & $\omega(C)=0.7486$ & $\omega(U)=0.1399$ & $\omega(\mathrm{UN})=0.1115$ \\
First pattern & $\omega(C)=0.7909$ & $\omega(M)=0.0703$ & $\omega(\mathrm{UN})=0.1389$ \\
Second pattern & $\omega(U)=0.0013$ & $\omega(M)=0.4328$ & $\omega(\mathrm{UN})=0.5659$ \\
\hline
\end{tabular}

TABLE 6: The diagnostic results of various states with the defect degrees by the proposed method.

\begin{tabular}{|c|c|c|c|c|c|c|c|c|c|}
\hline \multirow{2}{*}{ Test state } & \multirow{2}{*}{ Defect degree } & \multicolumn{3}{|c|}{ Simple diagnosis (first step) } & \multicolumn{4}{|c|}{ Precision diagnosis (second step) } & \multirow{2}{*}{ Judge state } \\
\hline & & $\omega(N)$ & $\omega(A)$ & $\omega(\mathrm{UN})$ & $\omega(C)$ & $\omega(U)$ & $\omega(M)$ & $\omega(\mathrm{UN})$ & \\
\hline Normal $(N)$ & & 0.8558 & 0.0724 & 0.0718 & & & & & $N$ \\
\hline \multirow{3}{*}{ Cavitation $(C)$} & Mild & 0.3529 & 0.6038 & 0.0433 & 0.7382 & 0.0579 & 0.1763 & 0.0276 & $C$ \\
\hline & Medium & 0.2311 & 0.7621 & 0.0068 & 0.9218 & 0.0053 & 0.0204 & 0.0525 & $C$ \\
\hline & Severe & 0.3200 & 0.6134 & 0.0666 & 0.9649 & 0.0001 & 0.0098 & 0.0252 & C \\
\hline \multirow{3}{*}{$\begin{array}{l}\text { Impeller } \\
\text { unbalance }(U)\end{array}$} & Mild & 0.2026 & 0.6813 & 0.1161 & 0.0153 & 0.7468 & 0.1934 & 0.0445 & $U$ \\
\hline & Medium & 0.1897 & 0.7385 & 0.0718 & 0.0118 & 0.8216 & 0.1430 & 0.0236 & $U$ \\
\hline & Severe & 0.1365 & 0.7027 & 0.1608 & 0.0092 & 0.7604 & 0.2259 & 0.0045 & $U$ \\
\hline \multirow{3}{*}{$\begin{array}{l}\text { Shaft } \\
\text { misalignment } \\
(M)\end{array}$} & Mild & 0.3415 & 0.6435 & 0.0150 & 0.1138 & 0.2126 & 0.6072 & 0.0664 & $M$ \\
\hline & Medium & 0.2071 & 0.7446 & 0.0483 & 0.0158 & 0.1901 & 0.6831 & 0.1110 & $M$ \\
\hline & Severe & 0.3007 & 0.6756 & 0.0237 & 0.0576 & 0.1573 & 0.6315 & 0.1536 & $M$ \\
\hline
\end{tabular}


TABLE 7: The diagnostic results of various states with the defect degrees by fuzzy neural network.

\begin{tabular}{|c|c|c|c|}
\hline \multirow{2}{*}{ Test state } & \multirow{2}{*}{ Defect degree } & \multicolumn{2}{|c|}{ Judge state by fuzzy neural network } \\
\hline & & Direct diagnosis & Sequential diagnosis \\
\hline Normal $(N)$ & & $N$ & $N$ \\
\hline \multirow{3}{*}{ Cavitation $(C)$} & Mild & $N$ & $N$ \\
\hline & Medium & $N$ & C \\
\hline & Severe & C & C \\
\hline \multirow{3}{*}{ Impeller unbalance $(U)$} & Mild & $N$ & $U$ \\
\hline & Medium & $U$ & $U$ \\
\hline & Severe & $U$ & $U$ \\
\hline \multirow{3}{*}{ Shaft misalignment $(M)$} & Mild & $N$ & C \\
\hline & Medium & C & $M$ \\
\hline & Severe & $M$ & $M$ \\
\hline
\end{tabular}

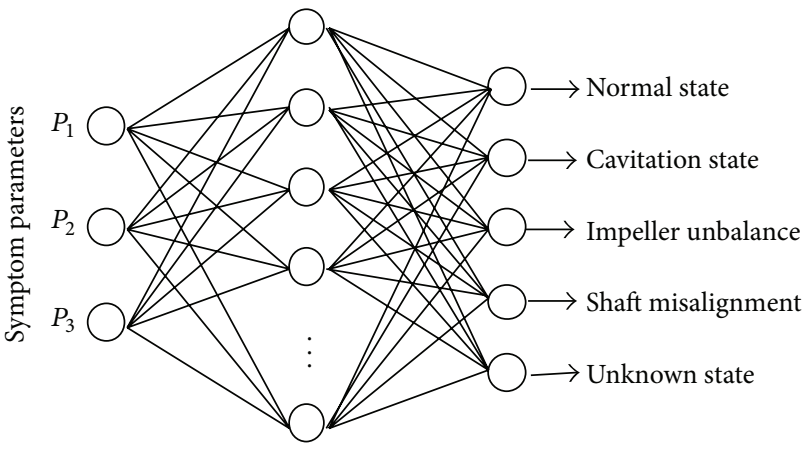

Input layer Middle layer Output layer

FIGURE 14: Direct diagnosis method based on fuzzy neural network.

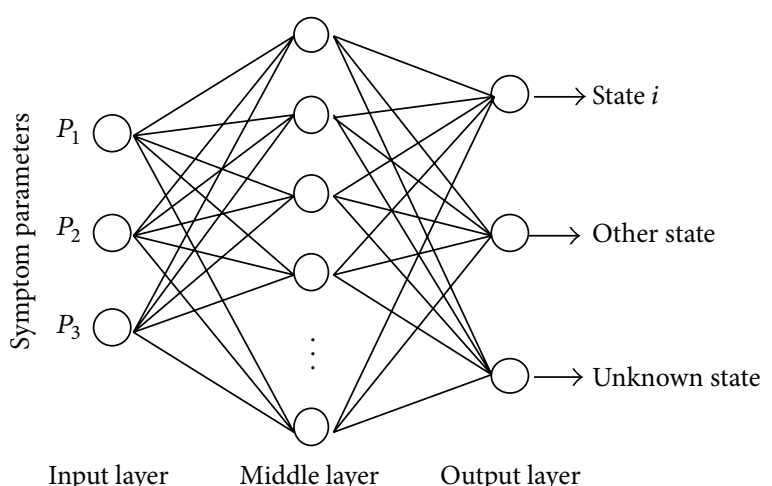

Figure 15: Sequential diagnosis method based on fuzzy neural network.

(2) Soft margin SVM is used to define synthetic symptom parameter (SSP) and then to raise the discernment sensitivity of original SPs.

(3) The efficiency of intelligent fuzzy diagnosis proposed here has been verified by applying it to a practical diagnosis for incipient faults of the centrifugal pump, with a performance. In fact, the perfect performance of intelligent diagnosis using possibility functions of the SSPs is attributed primarily to effective feature extraction by statistic filter, SVMs' generalization capability, SSPs' high sensitivity, and DST's combining evidence.

In the near future, the method proposed in this paper will be applied to condition diagnosis in various types of rotating machinery in a real plant.

\section{Conflict of Interests}

The authors declare that there is no conflict of interests regarding the publication of this paper.

\section{Acknowledgments}

This project is supported by the National Natural Science Foundation of China (Grant no. 51375037), National Program on Key Basic Research Project (Grant no. 2012CB026000), and Program for New Century Excellent Talents in University (NCET-12-0759).

\section{References}

[1] D. Moshou, I. Gravalos, D. Kateris, N. Sawalhi, and S. Loutridis, "Condition monitoring in centrifugal irrigation pumps with self-organizing feature visualisation," in Proceedings of the EFITA/WCCA Conference, pp. 116-124, 2011.

[2] S. Perovic, P. J. Unsworth, and E. H. Higham, "Fuzzy logic system to detect pump faults from motor current spectra," in Industry Applications Conference, vol. 1, pp. 274-280, Chicago, Ill, USA, 2001.

[3] C. S. Kallesøe, V. Cocquempot, and R. Izadi-Zamanabadi, "Model based fault detection in a centrifugal pump application," IEEE Transactions on Control Systems Technology, vol. 14, no. 2, pp. 204-215, 2006.

[4] S. Rajakarunakaran, P. Venkumar, D. Devaraj, and K. S. P. Rao, "Artificial neural network approach for fault detection in rotary system," Applied Soft Computing Journal, vol. 8, no. 1, pp. 740748, 2008.

[5] P. Jayaswal, A. K. Wadhwani, and K. B. Mulchandani, "Machine fault signature analysis," International Journal of Rotating Machinery, vol. 2008, Article ID 583982, 10 pages, 2008. 
[6] P. Brian, "Graney: pump vibration analysis," Pumps \& Systems, vol. 11, pp. 24-28, 2011.

[7] J. T. Renwick and P. E. Babson, "Vibration analysis, a proven techniques as a productions maintenance tool," IEEE Transactions on Industry Applications, vol. 21, no. 2, pp. 324-332, 1985.

[8] B. Liu and S.-F. Ling, "On the selection of informative wavelets for machinery diagnosis," Mechanical Systems and Signal Processing, vol. 13, no. 1, pp. 145-162, 1999.

[9] J. Lin and L. Qu, "Feature extraction based on morlet wavelet and its application for mechanical fault diagnosis," Journal of Sound and Vibration, vol. 234, no. 1, pp. 135-148, 2000.

[10] Q. B. Zhu, "Gear fault diagnosis system based on wavelet neural networks," Dynamics of Continuous Discrete and Impulsive Systems, Series A: Mathematical Analysis, vol. 13, part 2, pp. 671673, 2006.

[11] H. Wang, P. Chen, and S. Wang, "Intelligent diagnosis methods for plant machinery," Frontiers of Mechanical Engineering in China, vol. 5, no. 1, pp. 118-124, 2010.

[12] H. Wang and P. Chen, "Fuzzy diagnosis method for rotating machinery in variable rotating speed," IEEE Sensors Journal, vol. 11, no. 1, pp. 23-34, 2011.

[13] A. Albraik, F. Althobiani, F. Gu, and A. Ball, "Diagnosis of centrifugal pump faults using vibration methods," Journal of Physics: Conference Series, vol. 364, no. 1, Article ID 012139, 2012.

[14] H. Wang and P. Chen, "Intelligent diagnosis method for a centrifugal pump using features of vibration signals," Neural Computing and Applications, vol. 18, no. 4, pp. 397-405, 2009.

[15] K. Li, P. Chen, and S. Wang, "An intelligent diagnosis method for rotating machinery using least squares mapping and a fuzzy neural network," Sensors, vol. 12, no. 5, pp. 5919-5939, 2012.

[16] H. Wang and P. Chen, "Intelligent diagnosis method for rolling element bearing faults using possibility theory and neural network," Computers and Industrial Engineering, vol. 60, no. 4, pp. 511-518, 2011.

[17] A. Widodo and B.-S. Yang, "Application of nonlinear feature extraction and support vector machines for fault diagnosis of induction motors," Expert Systems with Applications, vol. 33, no. 1, pp. 241-250, 2007.

[18] H. T. Xue, "Structural fault diagnosis of rotating machinery based on distinctive frequency components and support vector machines," in Advanced Intelligent Computing Theories and Applications. With Aspects of Artificial Intelligence, vol. 6839 of Lecture Notes in Computer Science, pp. 341-348, 2012.

[19] A. Widodo and B.-S. Yang, "Support vector machine in machine condition monitoring and fault diagnosis," Mechanical Systems and Signal Processing, vol. 21, no. 6, pp. 2560-2574, 2007.

[20] H. Xue, H. Wang, P. Chen, K. Li, and L. Song, "Automatic diagnosis method for structural fault of rotating machinery based on distinctive frequency components and support vector machines under varied operating conditions," Neurocomputing, vol. 116, pp. 326-335, 2013.

[21] H. Xue, K. Li, H. Wang, and P. Chen, "Sequential diagnosis method for rotating machinery using support vector machines and possibility theory," in Intelligent Computing Theories, vol. 7995 of Lecture Notes in Computer Science, pp. 315-324, 2013.

[22] J. C. Principe and T. Yoon, "A new algorithm for the detection of tool breakage in milling," International Journal of Machine Tools and Manufacture, vol. 31, no. 4, pp. 443-454, 1991.

[23] M. Karimi-Ghartemani and M. R. Iravani, "A nonlinear adaptive filter for online signal analysis in power systems: applications," IEEE Transactions on Power Delivery, vol. 17, no. 2, pp. 617-622, 2002.
[24] W. Zhou, B. Lu, T. G. Habetler, and R. G. Harley, "Incipient bearing fault detection via motor stator current noise cancellation using wiener filter," IEEE Transactions on Industry Applications, vol. 45, no. 4, pp. 1309-1317, 2009.

[25] A. J. Volponi, H. DePold, R. Ganguli, and C. Daguang, "The use of kalman filter and neural network methodologies in gas turbine performance diagnostics: a comparative study," Journal of Engineering for Gas Turbines and Power, vol. 125, no. 4, pp. 917-924, 2003.

[26] C. M. Bishop, Neural Networks for Pattern Recognition, Oxford University Pres, New York, NY, USA, 1995.

[27] M. Čudina, "Detection of cavitation phenomenon in a centrifugal pump using audible sound," Mechanical Systems and Signal Processing, vol. 17, no. 6, pp. 1335-1347, 2003.

[28] L. Feinstein, D. Schnackenberg, R. Balupari, and D. Kindred, "Statistical approaches to DDoS attack detection and response," in Proceedings of the DARPA Information Survivability Conference and Exposition, vol. 1, pp. 303-314, 2003.

[29] T. Toyota, P. Chen, and T. Mizota, "Detection and distinction of failure signal by means of statistical tests of spectrum," The Journal of the Japan Society for Precision Engineering, vol. 58, no. 6, pp. 1041-1046, 1992.

[30] P. Chen, T. Toyota, and Z. He, "Automated function generation of symptom parameters and application to fault diagnosis of machinery under variable operating conditions," IEEE Transactions on Systems, Man, and Cybernetics Part A:Systems and Humans., vol. 31, no. 6, pp. 775-781, 2001.

[31] K. Li and P. Chen, "Intelligent method for diagnosing structural faults of rotating machinery using ant colony optimization," Sensors, vol. 11, no. 4, pp. 4009-4029, 2011.

[32] S. G. Mallat, "Theory for multiresolution signal decomposition: the wavelet representation," IEEE Transactions on Pattern Analysis and Machine Intelligence, vol. 11, no. 7, pp. 674-693, 1989.

[33] S. R. Gunn, "Support vector machines for classification and regression," Tech. Rep., University of Southampton, 1998.

[34] C. Cortes and V. Vapnik, "Support-vector networks," Machine Learning, vol. 20, no. 3, pp. 273-297, 1995.

[35] H. Matuyama, "Diagnosis algorithm," Journal of JSPE, vol. 75, no. 3, pp. 35-37, 1991.

[36] J. J. Richardson, Artificial Intelligence in Maintenance, Noyes Publications, New Jersey, NJ, USA, 1985.

[37] L. A. Zadeh, "Fuzzy sets," Information and Computation, vol. 8, no. 3, pp. 338-353, 1965.

[38] D. Dubois and H. Prade, Possibility Theory: An Approach to Computerized Processing of Uncertainty, Plenum Press, New York, NY, USA, 1988.

[39] P. Chen and T. Toyota, "Method of failure detection by possibility theory and dempster \& shafer probability theory," Journal of Kitakyushu Medical and Engineering Cooperative Association, vol. 9, pp. 1-4, 1998.

[40] P. Chen and T. Toyota, "Sequential fuzzy diagnosis for plant machinery," JSME International Journal, Series C: Mechanical Systems, Machine Elements and Manufacturing, vol. 46, no. 3, pp. 1121-1129, 2003.

[41] A. P. Dempster, "Upper and lower probabilities induced by a multivalued mapping," The Annals of Mathematical Statistics, vol. 38, pp. 325-339, 1967.

[42] G. Shafer, A Mathematical Theory of Evidence, Princeton University Press, 1976. 
[43] P. Chen, Foundation and Application of Condition Diagnosis Technology for Rotating Machinery, Sankeisha Press, Nagoya, Japan, 2009.

[44] C. R. Parikh, M. J. Pont, and N. Barrie Jones, "Application of Dempster-Shafer theory in condition monitoring applications: a case study," Pattern Recognition Letters, vol. 22, no. 6-7, pp. 777-785, 2001.

[45] S. Schocken and R. A. Hummel, "On the use of the Dempster Shafer model in information indexing and retrieval applications," International Journal of Man-Machine Studies, vol. 39, no. 5, pp. 843-879, 1993. 

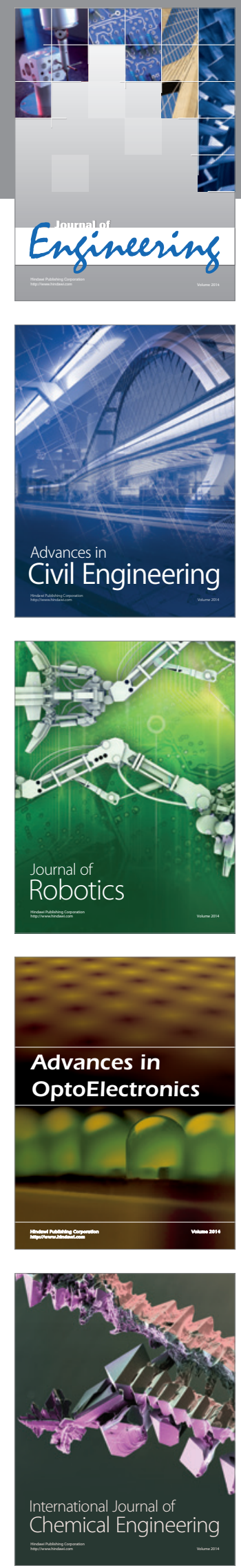

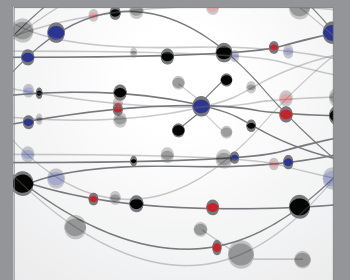

The Scientific World Journal
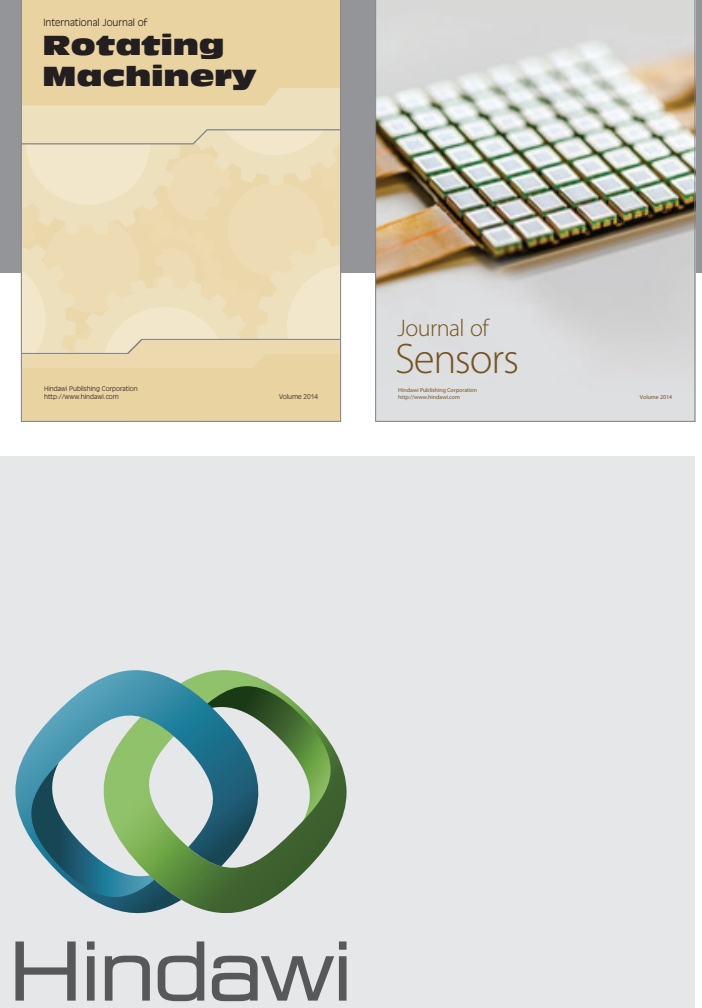

Submit your manuscripts at http://www.hindawi.com
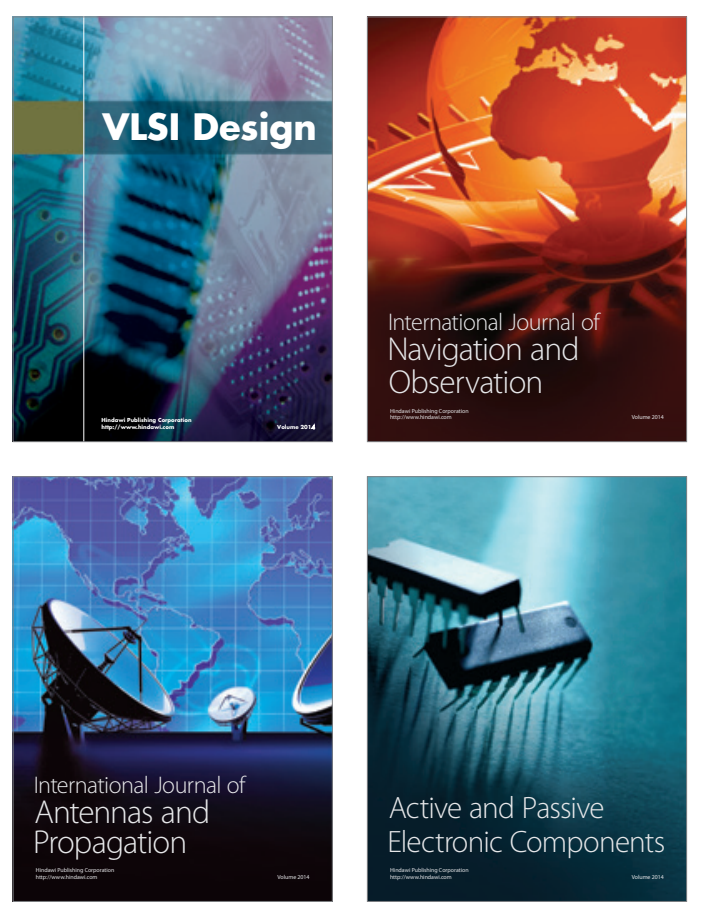
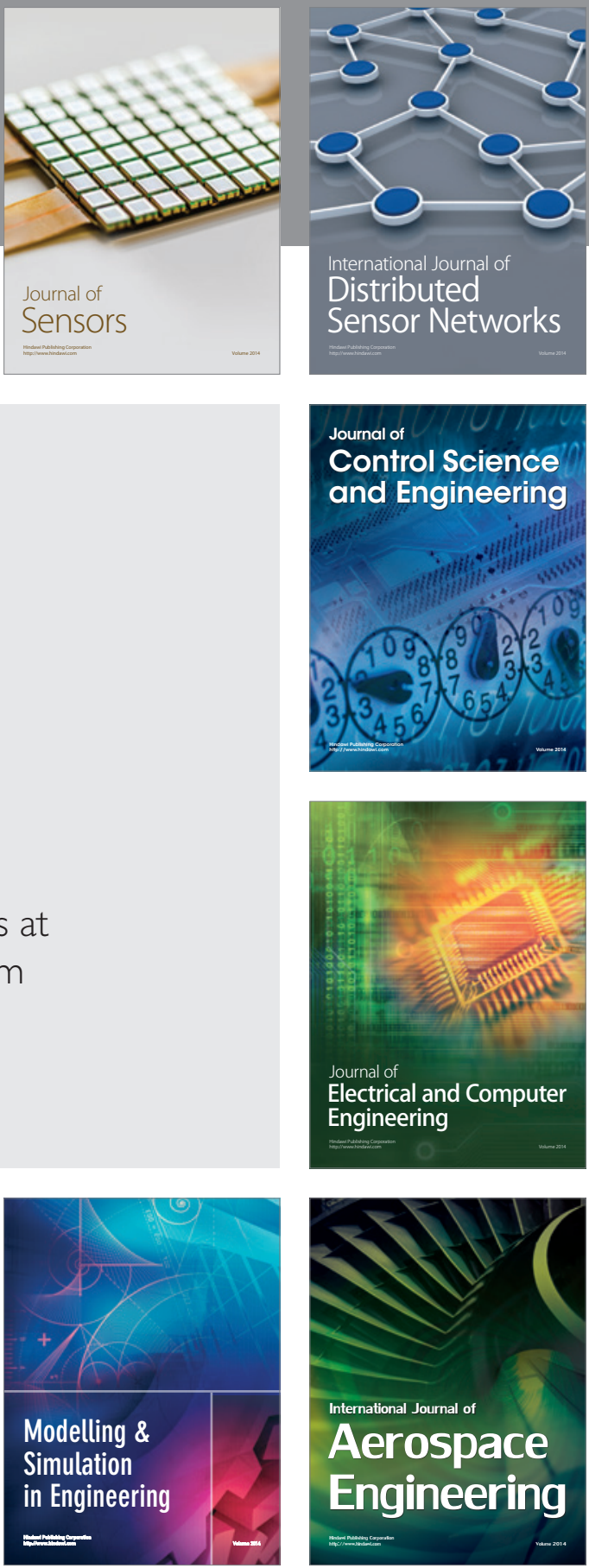

Journal of

Control Science

and Engineering
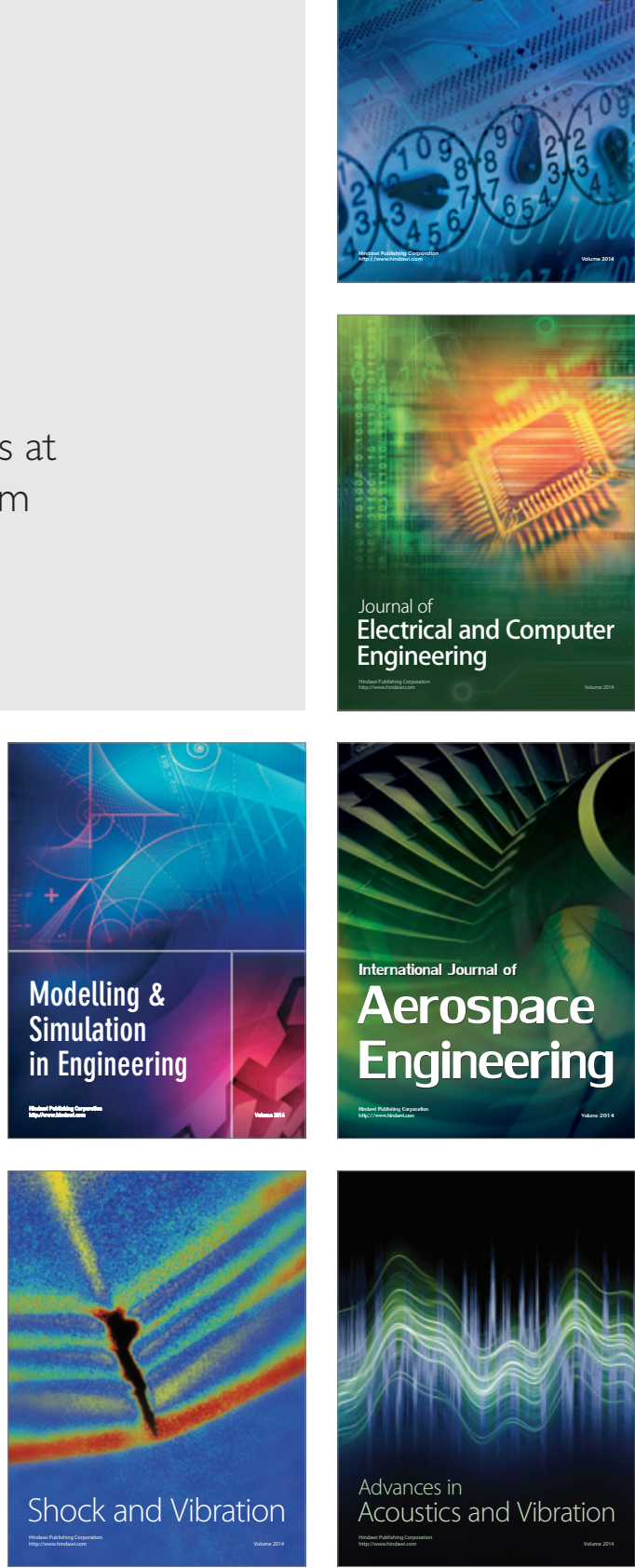